barrier heights (much less than $1 \mathrm{eV}$ ) were sometimes measured. The explanation ${ }^{4}$ is that forces could be acting between the tip and the surface which cause local elastic deformation and hence the tunnel gap movements to differ from the applied movements of the piezoelectric drive controlling the gap. These forces also have an effect on scaling in the microscope images. The idea of tip-to-surface forces acting in the scanning tunnelling microscope led Binnig, Quate and Gerber ${ }^{5}$ to suggest and to demonstrate the atomic force microscope. The principle of operation is similar to the scanning tunnelling microscope, but instead of tracing out contours of constant tunnel current, the microscope tip follows contours of constant force between tip and surface. The atomic force microscope thus closely resembles the traditional stylus profilometer, but the forces involved are several orders of magnitude smaller, in the region of $10^{-9} \mathrm{~N}$; thus the area of interaction between tip and surface is very small. This low level of force is sensed by the deflections of a very soft spring on which the tip is mounted. There is evidence that at these extremely low loads, no permanent (plastic) deformation occurs to the surface, even on the single-atom scale, and therefore with a small enough contact area, atomic resolution may be possible.

In their first results ${ }^{5}$, Binnig et al. demonstrate a lateral resolution of about $30 \AA$ A. Recently (Ch. Gerber, personal communication) they have imaged the atomic structure of graphite, which suggests that they have attained a lateral resolution of about $2-3 \AA$. Resolution normal to the surface is, as for the scanning tunnelling microscope, in the subangstrom range. These results are important because the atomic force microscope overcomes one of the main limitations of scanning tunnelling microscopy - that the surface to be studied must be conducting and completely free of insulating contamination. Provided a good understanding of the mechanics of the tip-surface interaction can be obtained, in particular the role of longer-range surface forces and the types of deformation present, then a whole new field of study is opened up. Ceramics, insulating and other surface films, and biological materials of unknown conductivity (the usual case on the single-molecule scale) are a few of the possibilities.

1. Binnig, G., Rohrer, H., Gerber, Ch. \& Weibel, E. Phys Rev. Lett. 50, 120-124 (1983).

2. Hamers, R.J., Tromp, R.M. \& Demuth, J.E. Phys. Rev. Lett. 56, 1972-1976 (1986)
Hamers, R.J., Tromp, R.M.

3. Becker, R.S., Golovchenko, J.A., \& Schwarzentruber, B.S. Nature 325, 419-421 (1987).

4. Coombs, J.H., \& Pethica, J.B. IBM J. Res. Dev. 30, $455-$ $459(1986)$.

5. Binnig, G., Quate, C.F. \& Gerber, Ch. Phys. Rev. Lett. 56 930-933(1986)

John Pethica is in the Department of Physics, University of Cambridge, Cavendish Laboratory, Cambridge CB3OHE, UK.

\section{Another cyclic-nucleotide-gated conductance}

UNDERSTANDING olfactory transduction has been a sticky problem. As recently reviewed in News and Views ${ }^{1}$, modern molecular and electrophysiological techniques are beginning to reveal details of the mechanism. On page 442 of this issue ${ }^{2}$, Nakamura and Gold report that isolated membrane patches pulled from the cilia of olfactory receptor cells have a conductance that is gated open, apparently directly, by cyclic GMP or cyclic AMP. It seems probable that this conductance is the link between odorant-stimulated cyclase(s) in olfactory cells ${ }^{3}$ and the odorant-sensitive current (see figure), and indicates a close parallel between olfactory and visual transduction.

This discovery brings to four the number of distinct conductances that seem to be activated directly by cyclic nucleotides. Fesenko et al. first discovered ${ }^{4}$ this type of conductance in patches of plasma membrane from the outer segments of vertebrate rod photoreceptors, so providing the long-sought-for link between the cyclic-GMP cascade and the light-sensitive membrane current in rods, and completing the picture of the excitatory mechanism of phototransduction. Conductances gated by cyclic

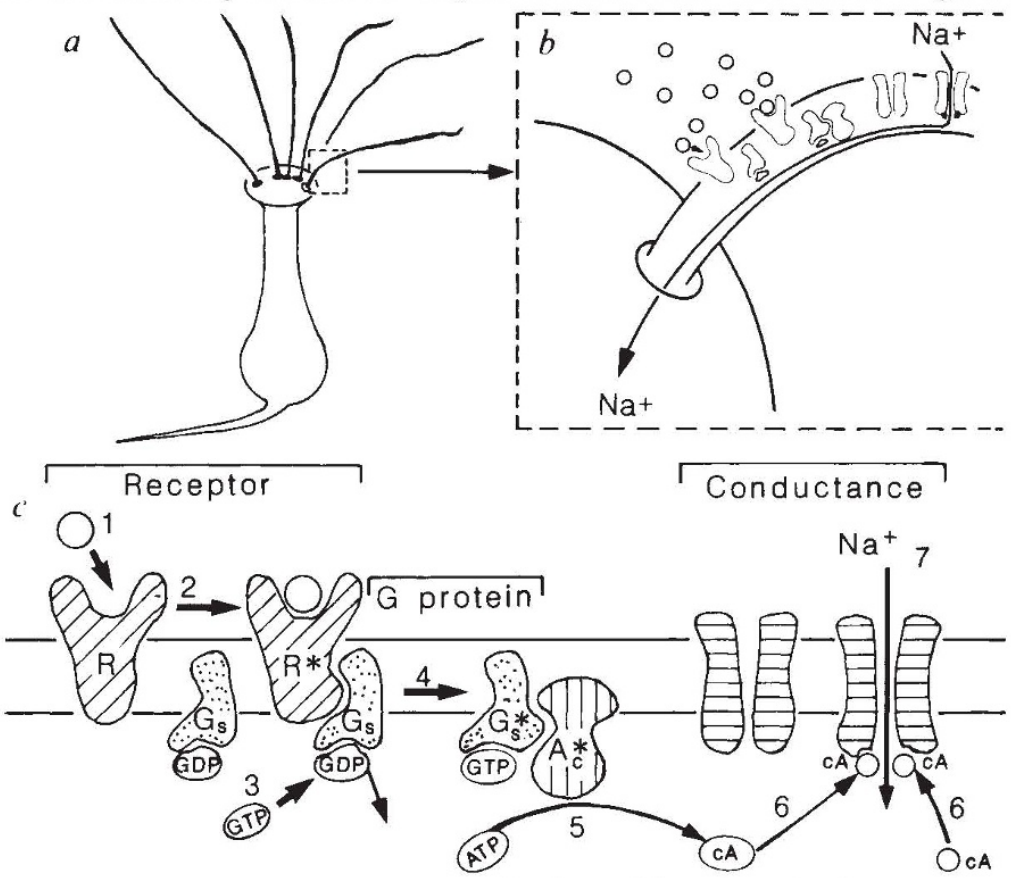

$a$, The olfactory cilia bear receptor molecules and ion channels, shown enlarged in $b . c$, Biochemical reactions triggered by binding of olfactant molecule to the receptor $(R)$, which converts it to active $R^{*}(2)$. This binds to the $G$ protein $\left(G_{s}\right)$ causing phosphorylation of GDP to GTP (3) and converting $G_{s}$ to $G_{s}^{*}$, which activates adenylate cyclase $\left(A_{c}^{*}\right)(4)$ and promotes conversion of ATP to cyclic AMP (cA) (5). Cyclic AMP binds to and opens the conductance (6), allowing an inward current to flow, subsequently depolarizing the sensory cell (see $b$ ).

nucleotides have subsequently been found in cone photoreceptor outer segments ${ }^{5}$ and in the membranes of the ventral photoreceptors of the horseshoe crab Limulus ${ }^{6}$.

The four conductances are not, however, identical. The cone conductance is distinguished from that of the rods by its voltage dependence. The rod, cone and olfactory conductances are all very small, between 10 and $15 \mathrm{fS}$ in the presence of normal divalent cations. The Limulus conductance, on the other hand, resembles a normal ion channel with a unitary conductance of $35-50 \mathrm{pS}$. The rod and cone conductances exhibit channel-like behaviour only in the absence of divalent cations. One prominent feature distinguishes the olfactory conductance from the others: it seems almost equally responsive to cyclic AMP and cyclic GMP, and to a much lesser extent to cyclic $\mathrm{CMP}^{2}$, whereas the photoreceptor conductances all have strong selectivity for cyclic GMP and are relatively unresponsive to other nucleotides ${ }^{4,5}$.

The progenitor of the family of cyclic-nucleotide-gated conductances may have already been present when simple ciliated receptor cells became specialized for either light or olfactory reception. The similarities that are emerging between photoreceptor, olfactory and hormone receptor mechanisms, all of which are mediated by GTPbinding proteins, are stimulating physiological, biochemical and genetic advances in the analysis of sensory transduction processes. We can expect rapid progress in unravelling the coupling between receptor and effector in these systems that are mediated by GTP-binding proteins and modulated by cyclic nucleotides.
1. Shepherd, G.M., Get
Nakamura, T. \& Gold, G.H. Nature 325, $442-444$ (1987).
3. Pace, U. et al. Nature $316,255-258$ (1985).
4. Fesenko, E.E., Kolesnikov, S.S. \& Lyubarsky, A.L. Nature 313, 310-313 (1984).
5. Haynes, L.W. \& Yau, K.-W. Nature 317, 61-64 (1986)
6. Johnson, E.C. et al. Nature $324,468-470$ (1986). 